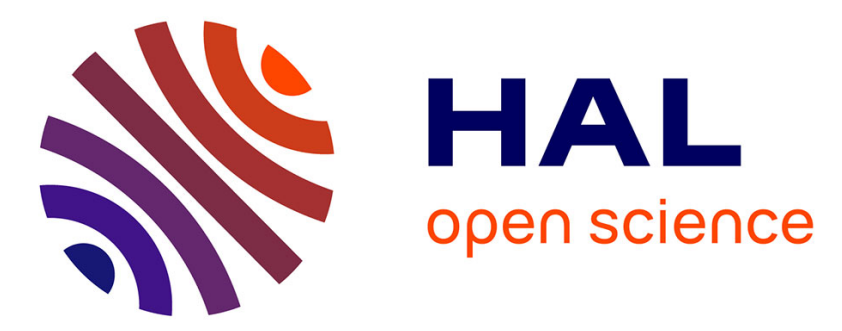

\title{
LE COMPLEXE D'ANTIGONE. RELECTURES FÉMINISTES ET POSTCOLONIALES DU SCÉNARIO OEDIPIEN
}

Crystel Pinçonnat

\section{> To cite this version:}

Crystel Pinçonnat. LE COMPLEXE D'ANTIGONE. RELECTURES FÉMINISTES ET POSTCOLONIALES DU SCÉNARIO OEDIPIEN. Revue de Litterature Comparee, 2012, Partages de l'Antiquité: les classiques grecs et latins et la littérature mondiale, 344, pp.495-509. hal-01382551

\section{HAL Id: hal-01382551 \\ https://hal.science/hal-01382551}

Submitted on 19 Oct 2016

HAL is a multi-disciplinary open access archive for the deposit and dissemination of scientific research documents, whether they are published or not. The documents may come from teaching and research institutions in France or abroad, or from public or private research centers.
L'archive ouverte pluridisciplinaire HAL, est destinée au dépôt et à la diffusion de documents scientifiques de niveau recherche, publiés ou non, émanant des établissements d'enseignement et de recherche français ou étrangers, des laboratoires publics ou privés. 


\title{
LE COMPLEXE D'ANTIGONE. RELECTURES FÉMINISTES ET POSTCOLONIALES DU SCÉNARIO OEDIPIEN
}

\author{
Crystel Pinçonnat
}

\section{Klincksieck | Revue de littérature comparée}

$2012 / 4-n^{\circ} 344$

pages 495 à 509

\section{ISSN 0035-1466}

Article disponible en ligne à l'adresse:

http://www.cairn.info/revue-de-litterature-comparee-2012-4-page-495.htm

Pour citer cet article :

Pinçonnat Crystel, « Le complexe d'Antigone. Relectures féministes et postcoloniales du scénario oedipien », Revue de littérature comparée, 2012/4 n³44, p. 495-509.

Distribution électronique Cairn.info pour Klincksieck.

(c) Klincksieck. Tous droits réservés pour tous pays.

La reproduction ou représentation de cet article, notamment par photocopie, n'est autorisée que dans les limites des conditions générales d'utilisation du site ou, le cas échéant, des conditions générales de la licence souscrite par votre établissement. Toute autre reproduction ou représentation, en tout ou partie, sous quelque forme et de quelque manière que ce soit, est interdite sauf accord préalable et écrit de l'éditeur, en dehors des cas prévus par la législation en vigueur en France. II est précisé que son stockage dans une base de données est également interdit. 


\section{Le complexe d'Antigone Relectures féministes et postcoloniales du scénario œdipien}

Les versions postcoloniales, africaines, francophones ou créoles d'Antigone ne manquent pas. Parmi celles-ci, on pourrait évoquer L'Antigone de l'Haïtien Félix Morisseau Leroy, joué pour la première fois en 1953 au RexThéâtre de Port-au-Prince. Comme il l'explique dans la préface, en adaptant en créole la pièce de Sophocle, le poète cherchait à faire une démonstration : « Nou kat milyon ayisyen ki pale kreyòl, nan pwen rezon pou'n pa aprann ekri'l

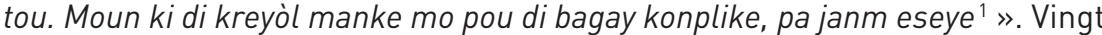
ans plus tard, Patrick Chamoiseau dédie, quant à lui, son adaptation d'Antigone à « tous nos fusillés assassinés d'oubli » et, en premier lieu, à Gérard Nouvet, lycéen de 17 ans tué pendant les émeutes de Fort-de-France en 1971. L'action de la pièce fait écho aux circonstances du drame. Dans la capitale martiniquaise, le préfet Créon interdit que l'on rende hommage au jeune homme et ordonne que sa dépouille pourrisse au soleil; une jeune fille passe outre l'arrêté pour honorer le cadavre. Écrouée sans procès, elle est surnommée Antigone par les lycéens. De telles adaptations, aux visées immédiatement politiques, ont tendance à susciter des lectures intertextuelles. Ces dernières adoptent généralement une démarche de type archéologique et privilégient les relations d'hypertexte à hypotexte, je m'intéresserai pour ma part à un tout autre travail du mythe, ce que j'appellerai, après d'autres, le complexe d'Antigone.

« Depuis la publication d'Antigone's Claim [Antigone : la parenté entre vie et mort], les réflexions philosophiques et féministes de Judith Butler nous ont invités à penser par delà CEdipe $»^{2}$. En 2000, dans son essai, la célèbre philo-

1. Félix Morisseau-Leroy, Antigone, Pétion-Ville (Haïti), Éd. Culture, 1953, p. 9; trad. «Nous sommes quatre millions d'Haïtiens à parler le créole, il n'y a aucune raison pour que nous n'apprenions pas aussi à l'écrire. Ceux qui disent que le créole manque de mots pour décrire des choses compliquées n'ont jamais essayé de le faire. »

2. Griselda Pollock, «Beyond CEdipus: Feminist Thought, Psychoanalysis, and Mythical Figurations of the Feminine », dans Vanda Zajko and Miriam Leonard (dir.), Laughing with Medusa. Classical Myth and Feminist Thought, Oxford, Oxford UP, 2006, p. 67-117, 
sophe avait en effet ébranlé les fondements de la construction freudienne en posant la question : «que serait-il arrivé si la psychanalyse avait pris Antigone et non CEdipe pour point de départ? $\gg .{ }^{3}$ À partir de cette réflexion, on tente aujourd'hui de repenser l'architecture freudienne du drame œdipien pour privilégier en son sein la figure d'Antigone et inverser la tendance historique qui, depuis la fin du XVIII e et le début du XIXe siècle, a voulu, selon George Steiner, qu' « CEdipe supplante Antigone $»^{4}$. Je souhaiterais poursuivre ce travail de révision et proposer une lecture de certains textes d'Assia Djebar, de Linda Lê et de Zahia Rahmani ${ }^{5}$, romancières d'expression française dont l'imaginaire est puissamment marqué du sceau de la colonisation. L'œuvre de chacune est hantée par le complexe d'Antigone; il donne corps à une condition historique aux retombées familiales tragiques.

\section{Le complexe d'Antigone}

La modernité a parfois eu tendance à réduire Antigone à un symbole de « résistance individuelle à l'arbitraire d'État au nom d'un devoir supérieur $^{6} »$. Dans The Antigone Complex, Cecilia Sjöholm pose un certain nombre de jalons qui permettent de dépasser cette approche étroitement politique. Premier point essentiel : «Antigone met en avant non pas une origine intelligible, mais plutôt un état de confusion. ${ }^{7} \mathrm{Ce}$ désordre tient au chevauchement généalogique dont elle est le fruit. Par là, comme on le verra dans les textes, Antigone défait toute tentative de raisonnement à partir de catégories duelles et antithétiques. En elle, les dichotomies s'effondrent: tant l'opposition entre les vivants et les morts, le féminin et le masculin, qu'entre ascendance et descendance. «Antigone est celle pour qui les positions symboliques sont devenues incohérentes, mélangeant comme elle le fait frère et père, surgissant comme elle le fait non comme une mère mais — l'étymologie le suggère “au lieu d'une mère" $\gg^{8}$. En outre, comme le sou-

p. 93 Ima traduction; je suis l'auteur des traductions pour lesquelles je ne donne pas de références).

3. Judith Butler, Antigone's Claim : Kinship between Life \& Death, New York, Columbia UP, 2000, p. 57; pour la traduction française de Guy Le Gaufey, Antigone : la parenté entre vie et mort, Paris, EPEL, 2003, p. 65.

4. George Steiner, Antigones [1984]; pour la traduction française de Philippe Blanchard, Les Antigones, Paris, Gallimard, 1986, p. 20.

5. Je remercie Christiane Chaulet Achour pour m'avoir aidée à bâtir le corpus de cet article. Je renvoie au beau texte où elle aborde la question : «Silence de père, écriture de fille. Le père "postcolonial" : Maïssa Bey et Zahia Rahmani », Christiane Chaulet Achour (dir.), Pères en textes - Médias et Littérature, Paris, Le Manuscrit, 2006, p. 103-115.

6. Rose Duroux et Stéphane Urdician, «Antigone, retours sur une fascination », Rose Duroux et Stéphane Urdician (dir.), Les Antigones contemporaines (de 1945 à nos jours), Clermont-Ferrand, Presses Universitaires Blaise Pascal, 2010, p. 13-32, p. 18.

7. Cecilia Sjöholm, The Antigone Complex. Ethics and the Invention of Feminine Desire, Stanford, Stanford UP, 2004.

8. J. Butler, Antigone : la parenté entre vie et mort, op. cit., p. 31. 
ligne encore J. Butler, son nom est à « interprét[er] comme “antigénération" $»^{9}$ : Antigone est celle qui arrête une transmission catastrophique en n'enfantant pas. Dans le même geste, elle rabat l'axe vertical de la généalogie sur l'axe horizontal de la fratrie; elle crée ainsi un cul-de-sac, dramatisant à l'extrême la structure familiale et politique, ce «croisement de relations, nœud dans un réseau. Mailles resserrées d'un filet ${ }^{10}$, soit le complexe où elle est prise.

Rigidifiée dans sa posture de « sœur absolue $»^{11}$, la surexposition d'Antigone a eu tendance à la banaliser. « Tout se passe comme si on croyait trop la connaître ${ }^{12}$, écrit fort justement Ariane Eissen. Pensée en termes de complexe - comme on parle du complexe d'CEdipe - Antigone redevient un matériau ductile. Elle dessine «moins une forme qu'un espace de variations continues ${ }^{13}$ qui autorise le déplacement des différents syntagmes en jeu, leur traitement par l'ellipse, voire leur renversement. À certains puristes, Antigone pourra paraître ainsi démembrée. J'aurai, pour ma part, une perspective inverse. Le complexe d'Antigone restaure au contraire la puissance qui faisait parfois défaut à une figure peu malléable, parce que comme sclérosée dans son intransigeance. Il oblige le lecteur à devenir actif et à procéder à un travail de remémoration qui ranime l'héroïne de ses cendres et grâce auquel, tel le phénix, elle prend à nouveau corps. Re/membering Antigone, tel pourrait être le titre de mon article en anglais, un titre qui permettrait de jouer sur deux acceptions du verbe to remember : « se rappeler », mais aussi « remembrer », au sens de « reformer, rassembler les morceaux épars d'un tout ». Certains textes d'A. Djebar, de L. Lê et de Z. Rahmani réveillent chez le lecteur la mémoire d'Antigone. Le travail qu'il effectue l'extrait du carcan de pierre où ses souvenirs l'avaient souvent pétrifiée. Elle retrouve ainsi une vigueur et une jeunesse dont elle a souvent été privée.

\section{Antigone chez Assia Djebar, une figuration du nœud de mémoire}

Le complexe d'Antigone ne s'inscrit pas de la même façon dans les trois $œ u v r e s$ que je me propose d'examiner. J'ai retenu les occurrences les plus manifestes que j'ai pu y trouver, ma proposition de lecture - qui demanderait une mise à l'épreuve plus approfondie, j'en conviens - ne se prétendant nullement exhaustive.

9. Ibid.

10. Claude Rabant, «Complexe d'CEdipe», Dictionnaire de la psychanalyse, Paris, Encyclopédia Universalis et Albin Michel, 1997, p. 527.

11. Michèle Dancourt, «Ismène la survivante, la sœur niée : de Sophocle à Ritsos », dans Florence Godeau et Wladimir Troubetzkoy (dir.), Fratries. Frères et sœurs dans la littérature et les arts de l'antiquité à nos jours, p. 33-44, p. 42.

12. Ariane Eissen, «Antigone sur la scène contemporaine: analyse d'un changement de paradigme », dans Rose Duroux et Stéphane Urdician (dir.), Les Antigones contemporaines (op. cit.), p. 63-73, p. 72.

13. Cf. Sigmund Freud, «Das Ich und das Es» (1923); pour la trad. française de S. Jankélévitch, « Le Moi et le ça », Essais de psychanalyse, Payot, 1963. 
Dans l'œuvre d'A. Djebar, le complexe d'Antigone s'ancre explicitement par le biais de références à l'héroïne. Dans L'Amour, la fantasia, une même scène est reprise plusieurs fois sous différents angles, elle présente une fillette, « bergère de treize ans » :

[...] la première fille des Amroune, elle que les cousins, les voisins, les alliés, les oncles paternels accusent de se prendre pour un quatrième mâle, en fuyant le douar et les soldats français, au lieu de se terrer comme les autres femelles!

[...] La voici orpheline du frère tombé, dans cette aube de l'été immobile; nouvelle Antigone pour l'adolescent étendu sur l'herbe, elle palpe, de ses doigts rougis de henné, le cadavre à demi dénudé. ${ }^{14}$

Dans Les Nuits de Strasbourg, la référence permet de nouer les différentes mémoires que la romancière enchevêtre dans la ville-frontière. Sur cette scène alsacienne marquée du sceau de la guerre, elle présente plusieurs couples qui, dans leur composition multiculturelle, sont tous habités par de profonds antagonismes historiques: conflit franco-algérien qui marque le couple de Thelja, l'Algérienne, et de François, l'Alsacien ; mémoire de l'holocauste qui hante Ève, la juive algérienne, face à Hans, l'Allemand et, de façon plus globale, hostilité prégnante des colonisés à l'égard des colons dans de nombreux couples (celui de Jacqueline et d'Ali, entre autres). À partir de ces différents personnages placés sur une scène historique où se surimpriment la mémoire de la Seconde Guerre mondiale et celle des conflits prussien et algérien, A. Djebar poursuit son analyse du legs colonial. Elle explore ainsi « l'héritage pervers qui court à travers les générations » ${ }^{15}$.

Sur ce fond, l'intertexte avec la tragédie de Sophocle explicite la volonté d'en finir avec une histoire qui perpétue indéfiniment une généalogie de la violence. Antigone est cité et commenté à l'occasion d'une scène qui rend compte du «dernier "filage" » de la pièce par une troupe composée de jeunes comédiens issus de l'immigration :

- En vérité, [...] Sophocle s'est trompé : Antigone ne se pendra pas dans sa tombe, Hémon [...] ne va pas se transpercer de sa lame en se nouant au cadavre de son aimée [...]. Ne gardons que les paroles du vieux Tirésias [...] quand il prédit :

Voici déjà la haine bouleversant toutes les villes qui auront vu leurs guerriers déchirés n'obtenir d'autres tombes que des chiens ou des fauves...!

Je vous le dis, ici : Djamila s'en va noblement vers la tombe. Tous les malheurs annoncés par le messager à Créon, pourquoi les mettre en scène? Il me semble à moi qu'Antigone reste vivante à jamais emmurée et que toute la chaîne de malheurs va nous sembler suspendue !...

14. Assia Djebar, L'Amour, la fantasia (1985), Albin Michel, 1995, p. 175, c'est moi qui souligne.

15. Mireille Rosello, Declining the Stereotype: Ethnicity and Representation in French Cultures, Hanover, UP of New England, 1998, p. 165. 
Antigone devient l'aube qui nous parle, l'espoir qui s'allume au fond du désespoir... ${ }^{16}$

Si la scène peut sembler secondaire dans le roman, la référence à la tragédie la désigne cependant comme une forme de noyau dur, décentré au sein de la construction romanesque. Elle éclaire obliquement tous les couples du roman au sein desquels les femmes choisissent de dépasser la haine historique à laquelle les voue leur généalogie et d'assumer leur amour pour un amant qui appartient au camp ennemi. En déployant la mémoire de chacun des protagonistes, A. Djebar inscrit massivement dans son texte « la haine bouleversant toutes les villes qui auront vu leurs guerriers déchirés n'obtenir d'autres tombes que des chiens ou des fauves ». Cependant, à travers l'imposante présence scénique d'Antigone au sein de l'architecture romanesque : « haute silhouette - "large, forte et illuminante" — », la romancière tente poétiquement d'arrêter l'amoncellement de cadavres qui a caractérisé le passé. Pour ce faire, elle transforme Antigone en une magnifique figure d'endeuillée, une figure de pleureuse orientale qui, plutôt que de déclencher par son sacrifice un nouveau cortège de morts, prend sur elle toute la douleur du monde. Silhouette toute de solitude «dressée au centre », « seule avec [s] es larmes » ${ }^{17}$, Antigone envahit l'espace de ses plaintes, sa voix déployant « un flux de lamentations en mélopée de douceur mélancolique ${ }^{18}$. La romancière installe sa présence à la moitié du roman - une silhouette comme encadrée et surélevée dans l'intrigue par les clichés que prend Ève, la photographe, qui suit le filage de la pièce. Sa figure éclaire les différents couples au sein desquels les femmes enfreignent les interdits amoureux dictés par leur filiation, parmi lesquelles Thelja, «fille d'un homme tué par l'armée française ${ }^{19}$. En partageant ses nuits avec François, Thelja conteste des lois qu'elle ressent comme masculines, mais sur lesquelles veillent jalousement les gardiennes de la tradition : « N'es-tu pas injuste, toi, une mère : comme au pays, tu veux nous appliquer leur loi, sur "nous", les femmes? $\gg^{20}$

Dans ce roman, A. Djebar produit une révision complète de l'hypotexte sophocléen. Pour comprendre le travail opéré, il faut retisser l'arabesque que dessine le texte. Thelja, l'Algérienne venue à Strasbourg, est hantée par un manuscrit du XII ${ }^{\mathrm{e}}$ siècle, l'Hortus deliciarum, « magnifique encyclopédie alsacienne qui demanda à son inspiratrice, la jeune Herrade, vingtcinq ans de sa vie, [...] de la prose latine parsemée d'emprunts en allemand souabe, [...] nombre de poèmes mystiques, inscrits en calligraphie gothique et illustrés par plus de centre trente cinq miniatures couleur ${ }^{21}$. Longtemps épargné, ce chef-d'œuvre fut détruit à jamais en août 1870 par les obus prussiens. On conçoit aisément l'idéal qu'incarne ce manuscrit tant pour Thelja

16. Assia Djebar, Les Nuits de Strasbourg, Paris, Actes Sud, 1997, p. 214-215.

17. Id., p. 212.

18. Id., p. 213.

19. Id., p. 244.

20. Id., p. 245.

21. Id., p. 101. 
qu'A. Djebar : œuvre hybride - tant linguistiquement que formellement produite par une femme, poétesse, dessinatrice et auteur de «chants grégoriens destinés au réconfort », il constitue une forme d'antithèse à l'art national qui célèbre les victoires coloniales, un art également évoqué dans le roman à travers le tableau d'Horace Vernet, « La prise de la Smala d'Abd el Kader » (1843), que Thelja voudrait faire découvrir aux jeunes comédiens. Face à ces deux voies : un art, objet de destruction, et un art qui fait de la destruction son objet, il est aisé de comprendre le choix qu'effectue A. Djebar. Pour arrêter le déferlement historique de la violence, la romancière prône une forme d'art qui lutte contre la destruction. Pour ce faire, elle forge un texte qui se donne à lire comme un rapt postcolonial, un geste artistique qui ne détruit pas, mais revivifie par hybridation sur le modèle de l'Hortus deliciarum. De façon symptomatique, la troupe de jeunes comédiens qui interprète Antigone prend pour nom « la Smala », un mot arabe passé en français qui, comme l'explicite le texte, « fait penser à un enlèvement, à un rapt, à un pillage de manuscrits précieux, de livres rares ${ }^{22}$. Pour ne pas relayer la violence guerrière que porte Antigone, la jeune troupe doit arracher le personnage éponyme à la loi qui le régit traditionnellement : la loi clanique. Dans leur interprétation, Antigone est « révoltée contre les siens, ayant coupé les amarres, dédaigneuse de la prétendue solidarité du groupe ${ }^{23}$. Chez eux, Antigone demeure une figure violente et intransigeante mais qui, à l'inverse de son modèle, ne se sacrifie pas aux siens. Elle choisit au contraire de jaillir de la tombe à laquelle la voue la tradition - tant littéraire que familiale et culturelle. « Jeune fille habillée de blanc », Djamila-Antigone se place audelà des conflits. Elle s'agrège à la mémoire de la ville et devient « l'une des plus belles statues de [la] cathédrale ». Elle rompt avec ceux de son clan et ose publiquement pleurer un amour doublement contre-nature selon leur loi : un amour pour une Française assassinée par l'un des siens.

Dans Les Nuits de Strasbourg, le complexe d'Antigone défait la forme conventionnelle du mythe. Dans ce texte conçu comme un «nœud de mémoire » où les passés de chacun se télescopent, montrant les « relais et ricochets entre différents lieux et différents temps ${ }^{24}$ qu'implique tout processus de remémoration, cette Antigone refuse la loi à laquelle les autres obéissent généralement. Par là, elle met un terme à la violence de guerre et à sa perpétuation, une violence aux figurations éparpillées tout au long du texte au gré des souvenirs de chaque personnage. Si cette violence n'a eu de cesse de se diffuser, d'essaimer et de migrer d'un lieu à l'autre, d'un contexte historique et géopolitique à un autre, la déploration d'Antigone l'absorbe. Érigée en statue qui domine la scène alsacienne, elle symbolise tous les êtres ayant reçu en héritage des blessures de guerre, sans distinction de patrie. Figure de deuil en majesté, sa verticalité l'emporte sur l'horizontalité

22. Id., p. 216.

23. Id., p. 329.

24. Michael Rothberg, «Writing Ruins: The Anachronistic Aesthetics of André SchwarzBart », dans R. Clifton Spargo et R. Ehrenreich (dir.), After Representation? The Holocaust, Literature, and Culture, New Brunswick, Rutgers UP, 2009, p. 99-118, p. 101. 
du gisant, même si elle contient en son sein « la face d'un proche aimé Imon père jamais connu ?) exposée là si près de ma main tremblante ${ }^{25}$.

\section{Linda Lê. Du complexe au paradoxe d'Antigone}

L'œuvre de Linda Lê est complexe, elle est cependant traversée par des motifs qui reviennent de façon obsessionnelle et qui, si on les tisse ensemble, donnent prise. Parmi ceux-ci : l'abandon du père, mort seul sans le secours de sa fille, et le souvenir de cette désertion lié à la perte du pays d'enfance qui ronge la narratrice de plusieurs textes. Cette configuration donne valeur de compensation à l'écriture, une «écriture [qui doit] tenir lieu de racines. $\gg^{26}$ Dans la grammaire personnelle que la romancière développe pour conjuguer ces données biographiques, Antigone tient une place de choix. Elle constitue un paradigme important pour « la métèque [...qui...] inventa une syntaxe à ses désarrois ${ }^{27}$. Dans Le Complexe de Caliban, ouvrage où l'écrivain commente certains textes fondateurs, un chapitre est intitulé «Antigone dans un paysage de cris». Ce dernier se nourrit de la lecture des Antigones de G. Steiner et, sur le même modèle que cette somme critique magistrale mais dans un autre format, il produit une glose de différents textes où s'inscrit l'héroïne. Texte qui s'alimente d'autres textes dans une forme de dévoration, «Antigone dans un paysage de cris » illustre l'un des principes qui traverse toute l'œuvre de L. Lê : «Mon livre, pour exister, avait mangé d'autres livres. ${ }^{28}$

Le complexe d'Antigone tel qu'il prend corps ici donne à voir cet acte cannibale où la digestion se fait également distorsion. Un deuil non accompli hante les textes de L. Lê. Il est souvent figuré sous la forme d'un fantôme ou d'un cadavre qui fait retour et menace de contaminer le règne des vivants. En ce sens, selon la belle formule de G. Steiner, chez elle comme dans Antigone, «la roue de l'être a effectué un tour obscène ${ }^{29}$. La cosmologie de la vie et de la mort a été inversée, faisant « de la vie une morte vivante et de la mort une survie organique désacralisée ${ }^{30}$. Lettre morte s'ouvre sur ces mots:

Les morts ne nous lâchent pas [...]. C'est le supplice de Mézence que j'endure, attachée à un mort, main contre main, bouche contre bouche, dans un triste embrassement. [...] Il me semble que je suis morte, tandis que mon père, ce mort qui ne me laisse pas en paix, déborde de vie. ${ }^{31}$

Le geste créateur tente généralement d'endiguer ce risque de nécrose, tout en le mettant en scène de façon exacerbée. La complexité du processus

25. A. Djebar, Les Nuits, op. cit., p. 52.

26. Linda Lê, Le Complexe de Caliban, Paris, Christian Bourgois, 2005, p. 33.

27. Id., p. 33-34.

28. Linda Lê, Les Évangiles du crime (1992), Paris, Christian Bourgois, 2007, p. 197.

29. G. Steiner, Les Antigones, op. cit., p. 314.

30. Ibid.

31. Linda Lê, Lettre morte, Paris, Christian Bourgois, 1999, p. 9, souligné par l'auteur. 
est bien décrite par Jack Yeager qui écrit : «le désir de faire scandale et de générer l'excès vient de la capture du sujet dans un réseau où l'incomplétude et le deuil liés à la perte de la langue et de la culture perdurent par delà toute nouvelle acquisition linguistique et culturelle. ${ }^{32}$

Si ce paradigme est infiniment décliné, L. Lê n'utilise pas cependant la notion de « complexe d'Antigone » pour le nommer. Dans son œuvre, comme l'indique le titre choisi pour son ouvrage, la notion de complexe est associée au personnage de Caliban. Par «complexe de Caliban», la romancière désigne ce dont souffre l'écrivain exilé : une «dévotion à la langue [de Prospéro, du maître] [...] mêlée d'hérésie »33 Autour d'Antigone, L. Lê forge, du même coup, une autre notion et parle en termes de «paradoxe ». Chez elle, l'héroïne a une double mission : lutter contre « le discours creux et le kitsch sanglant ${ }^{34}$, les deux fléaux qui traduisent, sur le plan discursif, la perte de l'objet d'amour et la violence hyperbolique qu'elle déclenche. Pour combattre ces « deux menaces [qui] pèsent sur le langage », Antigone doit « mourir à soi pour permettre la naissance du langage ${ }^{35}$; c'est là « le paradoxe d'Antigone ». Ce paradoxe génère un même scénario tragique : la mort libère non seulement l'héroïne de l'enfermement qu'elle subit, mais elle crée également une «ouverture ${ }^{36}$. Cronos, fable politique qui étouffe le lecteur de son «kitsch sanglant», déploie sans répit - presque sans blanc - le récit des crimes de Karaci, ministre de l'Intérieur du Grand Guide. Dans ses lettres, Una, jeune femme qui s'est sacrifiée pour sauver son père mais qui résiste à la flamme de Karaci, son époux, « comptabilise la créance de sang ${ }^{37}$. Elle tient les comptes pour toutes «les mères qui n'ont pu inhumer leurs fils selon la tradition $»^{38}$. Dans la ville comparée à un « immense ossuaire ${ }^{39}$, Una est tenue prisonnière. Aussi quand, à la fin du récit, elle va au supplice, acquiert-elle la grandeur d'une Antigone : «j'irai jusqu'au terme de mon calvaire, car alors mon sacrifice s'inscrira dans les mémoires. ${ }^{40}$ Sa lettre-testament ne sonne pas le glas de la défaite. Elle clôt le roman et laisse présager une ère nouvelle : «Nous sommes loin de l'épilogue, [...] demain les guetteurs verront se lever une nouvelle aube. ${ }^{41}$

La «grammaire de protestation contre l'oubli ${ }^{42}$ dont Antigone est ici porteuse est paradoxale non seulement parce qu'elle transforme la mort en victoire, mais aussi parce qu'elle défait l'étanchéité de catégories générale-

32. Jack A. Yeager, « Culture, Citizenship, Nation. The Narrative texts of Linda Lê », dans Alec G. Hargreaves et Mark McKinney, Post-colonial Cultures in France, New York, Routledge, 1997, p. 255-267, p. 263.

33. L. Lê, Le Complexe de Caliban, op. cit., p. 102.

34. Id., p. 157.

35. Id., p. 156.

36. Id., p. 161.

37. L. Lê, Cronos, op. cit., p. 71.

38. Id., p. 72.

39. Ibid.

40. Id., p. 163.

41. Id., p. 164.

42. Id., p. 153. 
ment pensées comme antithétiques. Dans Lettre morte, la narratrice évoque elle aussi une figure d'Antigone : depuis la mort de son père, «morte, exilée au Royaume de la Perte ${ }^{43}$, elle «voi[t] la vie comme d'un sous-sol ${ }^{44}$. À force d'être malaxé, le scénario traditionnel est cependant totalement retourné. «Le père, en mourant, est devenu le fils ${ }^{45}$, changement qui donne lieu au spectacle d'une Antigone en pietà. Chacune des composantes se voit radicalement renversée - le geste funéraire se fait déterrement, la mort et sa déploration résurrection :

Je rêve souvent que je me rends au bord du cours d'eau et que je déterre mon père. Je soulève la pierre tombale, je creuse la terre de mes mains, j'extrais du cercueil le cadavre que je prends dans mes bras. Il revient doucement à la vie. Il remue les lèvres, ouvre les yeux, me regarde et dit, Te voilà enfin. ${ }^{46}$

Chez L. Lê, on le voit, la figure d'Antigone se nourrit d'autres mythologies. Le paradoxe d'Antigone est d'autant plus plastique qu'il se greffe sur le complexe de Caliban. D'une part, au sein du triangle familial, Antigone doit se libérer de la figure dévoratrice du père mort, de ce « corps-à-corps avec un fantôme $\gg^{47}$ auquel la mère, en abandonnant le père, l'a vouée. D'autre part, elle doit refuser de devenir l'esclave d'une autre figure de la loi : celle du maître de la langue, un Prospéro faisant régner une dictature si froide ou un effroi si sanglant qu'il en vient à rejoindre certaines figurations de Créon. Antigone est ainsi sans cesse démembrée mais resurgit, à chaque fois, des quelques morceaux épars que le lecteur averti recueille comme la manifestation évanescente de sa présence toujours répétée. Si sa figure s'immisce souvent dans des récits de deuils tel Lettre morte, dans bien des cas, L. Lê la met en scène dans des textes comme Cronos qui sont autant de « concentrés de terreur » ${ }^{48}$. « Face aux mots de piété incandescente d'Antigone », inscriptions ténues qui témoignent de sa foi en l'écriture, une écriture qui l'emporte sur la sauvagerie malgré la mort, la romancière sature son texte des «mots de Créon, porteurs de mort » ${ }^{49}$. Tout en déclinant infiniment sur le plan narratif le paradoxe d'Antigone, chacun des textes de L. Lê rejoue sur le plan métapoétique l'éternel combat qui anime son écriture et qui la transforme en « une lame ouverte qui blesse et réveille le lecteur $\gg^{50}$. Pour que les mots aiguisés d'Antigone écorchent la chair du lecteur, il aura d'abord fallu créer une forme d'amoncellement de cadavres, de crimes et de mots. Au sein de cet empilement, une figure d'Antigone parvient à créer un habitacle, invagination mortuaire d'où renaît l'expression d'une intériorité, soit la figuration antithétique tant du « discours creux » que du « kitsch sanglant ».

43. Id., p. 19

44. L. Lê, Lettre morte, op. cit., p. 11.

45. Id., p. 26.

46. Id., p. 50 .

47. Id., p. 10.

48. L. Lê, Le Complexe de Caliban, op. cit., p. 156.

49. Ibid.

50. Id., p. 48. 


\section{Zahia Rahmani. Antigone ou la violence coloniale mise à nu}

Si, à ma connaissance, aucune référence explicite à Antigone n'intervient dans l'œuvre de Zahia Rahmani, l'intertexte sophocléen résonne cependant puissamment dans Moze, le beau texte que la romancière consacre à son père, un ancien harki mort le 11 novembre 1991, trouvé noyé dans l'étang communal après avoir salué le monument aux morts.

Bien qu'il s'agisse d'un récit, Moze adopte un modèle théâtral. S'y met en place l'architecture que Z. Rhamani reprendra dans «Musulman ». Roman (2005), dont la table des matières fait clairement apparaître cinq actes. Le mot «acte » n'est pas encore inscrit dans Moze, mais on y trouve néanmoins la même organisation : cinq parties précédées d'un prologue et bouclées par un épilogue. Si le lecteur ne prête pas forcément attention à cette construction dans un premier temps, elle n'est cependant nullement ornementale. Elle prend tout son sens dans certains passages dialogués qui évoquent un texte théâtral. C'est le cas des parties respectivement intitulées «II. La Sépulture » et «III. La Justice». Dans la première (sous-titrée «Les filles de Moze retournent dans le pays de leur père »), la narratrice et sa sœur sont de retour en Algérie pour tenter d'y inhumer le cadavre de leur père, frappé de bannissement. Elles s'affrontent mais, dépourvues de nom qui permettrait de les distinguer clairement, leurs répliques perdent peu à peu leur ancrage. L'échange évoque dès lors un texte comme Enfance de Nathalie Sarraute, un dialogue entre un même personnage scindé en deux instances d'énonciation distinctes qui s'opposent et se répondent.

Dans «La Justice » sous-titrée «La Fille de Moze est reçue par la Commission nationale de réparation », la narratrice est convoquée par la République française pour témoigner :

\section{- APPROCHEZ!}

Ainsi commence l'acte de justice.

Je suis dans un grand espace face à un haut mur. J'entends, approchez et je sais que je vais dire ce que je veux dire. Tout ce que je tiens à dire je vais le dire.

- Approchez.

- Je ne vous vois pas.

- Nous vous entendons.

Je lève la tête. Pourquoi si haut $?^{51}$

Du fait de ce début de scène, tout l'acte - si l'on conserve la métaphore théâtrale - est complètement déréalisé. Le décor est vidé, les personnages convertis en instances abstraites, données qui, pour un lecteur contemporain, évoquent la nudité de la scène tragique classique. La solennité du ton associée à la platitude du langage produit le même effet :

- Parlez librement, nous vous écoutons, me dit un homme.

- Je jure de dire toute la vérité. 
- C'est une commission, pas un tribunal. Nous avons pour mission de recueillir dans la transparence et l'équité votre témoignage concernant votre père. Vous n'avez pas à prêter serment.

- Je vais dire la vérité. [...] Sur votre convocation, vous dites, Nous tenterons, par des moyens qu'il restera à définir, de réparer cette injustice qui a fait de vos parents et de leurs descendants des individus dépossédés de leur dignité. Je suis donc ici pour dire ces hommes. Je dois parler de lui, de lui particulièrement. ${ }^{52}$

La narratrice prend le langage administratif à la lettre. À ses longues périodes qui mettent tout affect à distance, elle oppose une parole faite de phrases courtes et fermes, scandées par un rythme ternaire. Peu à peu son discours se fait toutefois plus intime, se transformant en un monologue, presque une plainte : «Mon père, je ne l'ai connu qu'absent. [...] Moze était mon père, un père que je n'ai pas eu. Un père qui ne l'était pas. ${ }^{53}$

Dans les deux parties qui envisagent la confrontation avec des instances nationales, algérienne et française, le texte renoue ainsi avec une forme de rehaussement tragique. Du fait de cette stratégie conjuguée aux motifs de «La Sépulture » et de «La Justice », titres des deux actes qui reprennent le diptyque sophocléen où s'affrontent successivement Antigone et Ismène, puis Antigone et Créon, les éléments disséminés au fil du texte (parmi lesquels la dédicace : «à la mémoire de mon frère Mokrane ») viennent s'agglutiner et dessinent une nouvelle version d'Antigone. L'usage du nom «Antigone » est évité et, avec lui, son effet d'usure; on ne conserve que la force du témoignage qui culmine dans la partie centrale de ce «plaidoyer-réquisitoire ${ }^{54}$. Malgré cette absence, peut-être grâce à elle, plus que dans les autres œuvres précédemment évoquées, certains passages de Moze sont habitées d'un souffle tragique. Cet effet tient au travail de dénuement auquel ont été livrées les différentes instances en jeu, travail qui transforme la parole de la narratrice en une voix solitaire, hors communitas, presque d'outre-tombe. De fait, le dernier acte du récit intitulé «Moze parle » et sous-titré «La voix de Moze glisse dans sa fille » renoue de ses stichomythies avec le dialogue tragique :

- Pourquoi n'as-tu rien dit?

- Je suis mort!

- Sans une plainte, sans un mot! Pourquoi?

- Je ne peux pas en parler.

- Essaie, essaie encore. [...] Ils disent que nous sommes tes enfants!

- J'ai honte de cette honte que je ne vous ai pas dite. [...] J'ai étouffé mes mots.

- Tu en es mort!

- Je me suis noyé.

- Tu n'existeras pas!

- Je ne suis pas seul. Ton frère est avec moi.

- Lui n'était pas sale.

52. Id., p. 108-109.

53. Id., p. 109.

54. C. Chaulet-Achour, art. cit., p. 111. 
- J'ai l'ai souillé.

- Et moi?

- Faut-il pourrir la terre de ce pays de toute notre pourriture $?^{55}$

Ce duel verbal entre le père et la fille clôt l'acte. Il montre la torsion qu'a subie la trame initiale. Comme dans le monde grec cependant, le cadavre pourrissant menace d'envahir le réel et l'imaginaire. Les morts tourmentés hantent sans relâche les vivants: «Lui il me doit de me quitter, de quitter mon esprit, il me doit de partir. Il me doit de ne plus revenir. Je suis venue ici pour m'en débarrasser! $\gg^{56}$

\section{Conclusion. De la transmission filiale en contexte colonial}

J'ai choisi de finir ma réflexion en m'appuyant sur Moze, car non seulement Z. Rahmani y met au point une écriture radicalement nouvelle, mais son texte, dans son intransigeance, dévoile en outre les raisons profondes de la prégnance du complexe d'Antigone chez les auteurs féminins postcoloniaux. Dans Moze, le complexe d'Antigone colle au plus près du texte sophocléen. Des commentaires qui portent sur la tragédie grecque pourraient lui être appliqués :

Son père, le roi, a perdu son éclat, sa brillance phallique. Elle en est le témoin, elle en prend le relais, non pour expier une faute comme un agneau sacrificiel mais pour prêter sa voix à une vérité. Or ce qu'elle a à dire, Créon estime qu'il est préférable de le taire. ${ }^{57}$

Comme chez L. Lê, la figure de Polynice demeure dans l'ombre; c'est celle du père qui l'emporte. «Banni sans nom ${ }^{58}$, il contamine toute la lignée de sa faute et interdit la scission salvatrice. Le symptôme de cette nécrose s'inscrit dans le titre, Moze, non pas un prénom arabe ou berbère, mais un nom inventé par l'auteur qui déclare: «m’est arrivé, fin janvier début février 1999, “Moze”, c'est-à-dire ce prénom, cette chose qui est je pense la contraction du prénom de mon père et du mien. ${ }^{59}$ Moze est la figuration de l'indistinction mortifère qui menace l'héritière. De son prénom, Zahia, comme digéré par le nom du père, ne reste que le « $z$ » de l'initiale : en Moze, elle est enclavée, presque déjà morte, comme le suggère la paronomase. Aussi doit-elle se libérer de cette emprise du mort, point tant en

55. Id., p. 179-180.

56. Z. Rahmani, Moze, op. cit., p. 85.

57. Françoise Duroux, Antigone encore. Les Femmes et la loi, Paris, côté-femmes, 1993, p. 102.

58. Zahia Rahmani, « Mulsulman». Roman, Paris, Sabine Wespieser Éditeur, 2005, p. 85

59. Zahia Rahmani, «Le "harki" comme spectre ou l'écriture du "déterrement" », Catherine Coquio (dir.), Retours du colonial? Disculpation et réhabilitation de l'histoire coloniale, Nantes, L'Atalante, 2008, p. 221-237, p. 229. 
l'enterrant qu'en publiant son histoire, une histoire arrachée au silence, comme déterrée :

Par l'écriture je sais que je l'expose et le réduis. Par l'écriture je me défais de lui et vous le remets. Mais je rappelle, étant sa fille, que je suis aussi ce qui est venu par lui et qui le continue. Un legs. Une exécution testamentaire ouverte par son salut aux morts. ${ }^{60}$

« Faire parler le trauma [paternel] pour s'en détacher $»^{61}$, telle est bien l'expérience décrite. «Donne une langue à Moze! » ${ }^{62}$, ordonne sa sœur à la narratrice. L'écriture restitue la parole de celui chez qui elle fut avortée. Elle produit une maïeutique libératrice qui a pour vertu de séparer les vivants et les morts. Celui que Janine Altounian nomme l' « écrivain-greffier » n'est plus englué dans l'autre et son histoire. En assurant l'« expertise du sinistre », il mène un travail de déliaison qui lui permet d'assumer son héritage, un objet par là détaché de lui. La filiation peut dès lors être envisagée comme un lien positif : l'écriture a non seulement créé du jeu, soit l'espace nécessaire au tressage du récit, elle a également permis à la romancière de restaurer l'intégralité de son nom, un nom et un prénom qui lui sont propres. Ils sont inscrits sur la jaquette de son livre, un livre par le biais duquel elle reconnaît les rapports de violence propres à l'histoire des siens et grâce auquel elle célèbre la dignité de sa dette envers eux. Selon la belle formule de J. Altounian, « devenant le scribe des mutiques qu'il porte en lui, l'héritier écrivain raconte leur histoire et, ce faisant, l'histoire du monde qui les a rendus muets ${ }^{63}$.

Sur le modèle sophocléen, les différents textes que j'ai examinés mettent en scène des " "communauté[s]" familiale[s] catastrophique[s] [qui] multiplie[nt] les ligatures mortelles $»^{64}$. En leur sein, une même caractéristique travaille l'interprétation d'Antigone: la contestation de l'héritage hégélien qui « semble supposer la séparabilité de la parenté et de l’État » ${ }^{65}$. Dans tous, la confusion généalogique est le fait de l'héritage colonial : il a éradiqué l’ordre ancien et brisé les liens de parenté sur lesquels il reposait. La colonisation a créé de nouveaux réseaux de fidélité, incompatibles avec ceux de la fratrie : elle a provoqué la « rupture de la chaîne des frères » ${ }^{66}$. En ce sens, l'ordre familial a été contaminé par le régime colonial et a produit un legs négatif qui, dans tous les textes, perturbe le rapport au père. La puissance de l'empire tient au fait qu'il n'intervient pas comme une structure plaquée, étatique, qui s'imposerait par la seule force - figuration caricatu-

60. Zahia Rahmani, Moze, op. cit., p. 24.

61. Janine Altounian, L'Intraduisible. Deuil, mémoire, transmission, Paris, Dunod, 2005, p. 70.

62. Z. Rahmani, Moze, op. cit., p. 97.

63. J. Altounian, L'Intraduisible., op. cit., p. 72.

64. M. Dancourt, «Ismène la survivante, la sœur niée : de Sophocle à Ritsos », art. cit., p. 34.

65. J. Butler, Antigone : la parenté entre vie et mort, op. cit., p. 13.

66. Zahia Rahmani, «Là où les mondes se touchent », Conférence donnée à l'École régionale des Beaux-Arts de Nantes, 3 mars 2009, http://vimeo.com/5143150, site consulté en oct. 2011. 
rale de Créon et de son pouvoir. Le legs colonial est chéri : il a pénétré l'être intime de chacun sous la forme de la langue du colon.

«Vivre ensemble nous tue, nous séparer est mortel » ${ }^{67}$. Telle est la double contrainte que les textes déclinent à l'envi. Tantôt, comme chez L. Lê, c'est la séparation qui l'emporte et condamne à jamais :

Le fantôme me dévore. J'appelle mon père, je le supplie de revenir à la vie [...] pour que je puisse [...] recueillir sur ses lèvres [...] le dernier mot de son amour. [...] C'est ce mot perdu qui manque à mon vocabulaire, qui fait que toutes mes phrases trébuchent, que mon monologue restera lettre morte. ${ }^{68}$

Tantôt, comme chez Z. Rahmani, c'est le corps du père harki inféodé à la France qui en vient à incarner un legs colonial encombrant, négatif. Homme à la parole empêchée ( « il n'était que ce débordement sans voix » ${ }^{69}$ ), toujours demeuré sans droit, il revient à sa fille de non seulement relayer sa plainte mais aussi de l'articuler et de la publier dans la langue du colon, celle de l'État français qui a «fabriqu[é] une armée de soldatmorts [sic] sans se soucier qu'ils étaient des hommes $\gg^{70}$. La France a fait d'eux et de leurs enfants des prisonniers, prisonniers de ce «pèremort » [sic] qui, toute sa vie durant, a attendu que justice soit rendue :

Il voulait un statut d'ancien combattant, une pension militaire, des papiers spéciaux, un droit d'inventaire pour ce qu'il avait sacrifié, ses terres, ses arbres, ses biens, ses bêtes et surtout un droit au repos pour oublier. ${ }^{71}$

Si la figure du harki, « un banni, un être indigne ${ }^{72}$, incarne de façon hyperbolique l'être privé de ses droits et de tout territoire, A. Djebar montre le caractère retors de tout pouvoir à l'égard des victimes de guerre. Dans L'Amour, la fantasia, elle fait entendre le récit d'une femme, une veuve, autre figure bafouée :

J'ai eu quatre hommes morts dans cette guerre. Mon mari et mes trois fils. [...]

Mon frère fut le cinquième... Lui, je l'ai ramené de la rivière. J'ai cherché partout son corps; je l'ai retrouvé. Il est enterré au cimetière! [...]

À l'indépendance, les gens de la ville ne m'ont rien donné. Il y avait un responsable, du nom de Allal : le jour où il a fui, pour monter au maquis, je l'avais caché quelque temps chez moi! [...]

67. Formulation de Jean-Pierre Caillot et Gérard Decherf reprise par Francine AndréFustier, Fronçoise Aubertel dans « La transmission psychique en souffrance », Alberto Eiguer et alii, Le Générationnel. Approches en thérapie familiale et psychanalyse, Dunod, 1997, p. 128.

68. A. Djebar, Lettre morte, op. cit., p. 57-58.

69. Id., p. 19.

70. Z. Rahmani, Moze, op. cit., p. 20.

71. Id., p. 53.

72. Id., p. 23. 
- O Allal, où est mon droit? [...] Donne-moi mon droit !

Ils ne m'ont rien donné... tu vois où j'habite maintenant, il m'a fallu donner de l'argent pour occuper cette cabane. ${ }^{73}$

Les femmes aussi réclament leur dû. Elles n'osent jamais toutefois faire entendre la violence qui a été faite à leur corps :

«Ma » question frémit, entêtée. [...] Dire le mot secret et arabe de « dommage », ou tout au moins de « blessure » : [...]

- Ma fille, y a-t-il eu « dommage »? [...]

L'une ou l'autre des aïeules posera la question [...]. La jeune femme, les cheveux recoiffés, ses yeux dans les yeux de la vieille, éparpille du sable brûlant sur toute parole : le viol, non dit, ne sera pas violé. Avalé. ${ }^{74}$

Le français, ce don du père, «langue paternelle », permet à A. Djebar de sortir du «droit de réserve ${ }^{75}$ et de dépasser les non-dits imposés par l'arabe. C'est en effet dans la langue du colonisateur que la romancière capte toutes les voix asphyxiées des femmes livrées à l'ennemi et la densité de leurs silences. L'ennemi, c'est bien souvent le soldat français, mais c'est aussi l'e'dou, «mot acerbe dans sa chair arabe » qui écorche de sa sonorité «l'atmosphère environnante » ${ }^{76} d$ 'une scène de hammam, un mot qui signifie «l'ennemi » et qui désigne, quand les femmes parlent entre elles, le mari. Si les espaces extérieurs sont dévolus à la lutte armée et au combat des hommes, les femmes, quant à elles, se sont retranchées dans les espaces symboliques de l'intériorité, des lieux où les conflits demeurent larvés, irrésolus. Ils fermentent au sein même des maisons où le patriarcat réaffirme d'autant plus brutalement sa puissance qu'il a été foulé aux pieds par le colonisateur. Ils s'immiscent même dans la moiteur du hammam où la langue des femmes, marquée du sceau historique de la violence, les fait pénétrer.

Confusion généalogique, confusion linguistique, chez les trois romancières, l'héritage colonial - comme tatoué au plus profond de chacun empêche la transmission généalogique de s'effectuer. "Les colonisés dev[enus] héritiers des colons $\gg{ }^{77}$, la figure du père ne peut que s'en trouver malmenée. À défaut de pouvoir léguer sa terre, sa langue et son histoire à sa fille, le père lui livre son cadavre, son seul bien, nouant ainsi pour elle une alliance indéfectible avec la mort. Elle ne pourra rompre cette union morbide qu'en faisant un détour par la langue adverse. Seule antidote à la nécrose, elle lui permet d'injecter de l'autre dans le même, de défaire ainsi la fusion et de se séparer, d'exister seule.

Crystel Pinçonnat

Université d'Aix-Marseille, CIELAM (EA 4235)

73. A.Djebar, L'Amour, la fantasia, op. cit., p. 279-280.

74. Id., p. 282-283, souligné par l'auteur.

75. Je reprends l'expression à Mireille Calle-Gruber (cf. Assia Djebar ou la résistance de l'écriture. Regards d'un écrivain algérien, Paris, Maisonneuve \& Larose, 2001, p. 79].

76. A. Djebar, Vaste est la prison, Paris, Albin Michel, 1995, p. 13-14.

77. M. Calle-Gruber, Assia Djebar ou la résistance de l'écriture, op. cit., p. 98. 